Invited Paper

\title{
Breaking the glass ceiling: hollow OmniGuide fibers
}

\author{
Steven G. Johnson, Mihai Ibanescu, M. Skorobogatiy, Ori Weisberg, Torkel D. Engeness, \\ Marin Soljačić, Steven A. Jacobs, J. D. Joannopoulos, and Yoel Fink \\ OmniGuide Communications, Inc.
}

\begin{abstract}
We argue that OmniGuide fibers, which guide light within a hollow core by concentric multilayer films having the property of omnidirectional reflection, have the potential to lift several physical limitations of silica fibers. We show how the strong confinement in OmniGuide fibers greatly suppresses the properties of the cladding materials: even if highly lossy and nonlinear materials are employed, both the intrinsic losses and nonlinearities of silica fibers can be surpassed by orders of magnitude. This feat, impossible to duplicate in an index-guided fiber with existing materials, would open up new regimes for long-distance propagation and dense wavelengthdivision multiplexing (DWDM). The OmniGuide-fiber modes bear a strong analogy to those of hollow metallic waveguides; from this analogy, we are able to derive several general scaling laws with core radius. Moreover, there is strong loss discrimination between guided modes, depending upon their degree of confinement in the hollow core: this allows large, ostensibly multi-mode cores to be used, with the lowest-loss $\mathrm{TE}_{01}$ mode propagating in an effectively single-mode fashion. Finally, because this $\mathrm{TE}_{01}$ mode is a cylindrically symmetrical ("azimuthally" polarized) singlet state, it is immune to polarization-mode dispersion (PMD), unlike the doubly-degenerate linearly-polarized modes in silica fibers that are vulnerable to birefringence.
\end{abstract}

Keywords: Fiber optics, Bragg reflectors, omnidirectional mirrors, photonic crystals

\section{INTRODUCTION}

Telecommunications has continued to push optical fibers towards ever-more demanding applications-such as high bit rates, dense wavelength-division multiplexing (DWDM), and long distances - and in response there has been renewed interest in alternative fiber designs to lift fundamental limitations of silica fibers. A particularly exciting departure from traditional fibers are fibers based on photonic band gaps, forbidden frequency ranges in periodic dielectric structures that can confine light even in low-index or hollow regions [1]. Two main classes of fibers have emerged using photonic band gaps: photonic-crystal "holey" fibers that use a two-dimensional transverse periodicity [2], and Bragg fibers* that use a one-dimensional periodicity of concentric rings [3-16]. In this paper, we study the propagation of light in a novel class of Bragg fibers: "OmniGuide" fibers with a hollow core, which use a multilayer cladding that exhibits omnidirectional reflection in the planar limit $[7,10,15-17]$.

In the following sections, we show how OmniGuide fibers bear strong resemblances to the hollow metallic waveguides that are used in the microwave regime, confining a set of guided modes almost entirely within the hollow core with similar field patterns and dispersion characteristics. Because of this strong confinement, we prove that radiation leakage, material absorption, and nonlinearities from the cladding layers can be suppressed by many orders of magnitude. (Additional imperfections were considered in [15].) Moreover, like hollow metallic waveguides, we show that there is substantial loss discrimination between a single lowest-loss mode, $\mathrm{TE}_{01}$, and other guided modes - this produces modal filtering that allows even a highly-multimode OmniGuide fiber to operate in an effectively single-mode fashion. Because this $\mathrm{TE}_{01}$ state is cylindrically symmetrical and non-degenerate, it has the additional benefit of immunity to polarization-mode dispersion (PMD) from fiber birefringence. In this way, we demonstrate that OmniGuide fibers have the potential to lift three major physical limitations on silica fibers: losses, nonlinearities, and PMD [18].

\footnotetext{
Correspondence to M. S.: maksim@omni-guide.com; phone 617-551-8444; fax 617-551-8445; http://www.omniguide.com; OmniGuide Communications, One Kendall Square, Building 100 \#3, Cambridge MA 02139.

* Bragg fibers should not be confused with fiber Bragg gratings- the former use a lateral index modulation for transverse optical confinement, while the latter use axial modulation for longitudinal confinement and other effects.
} 

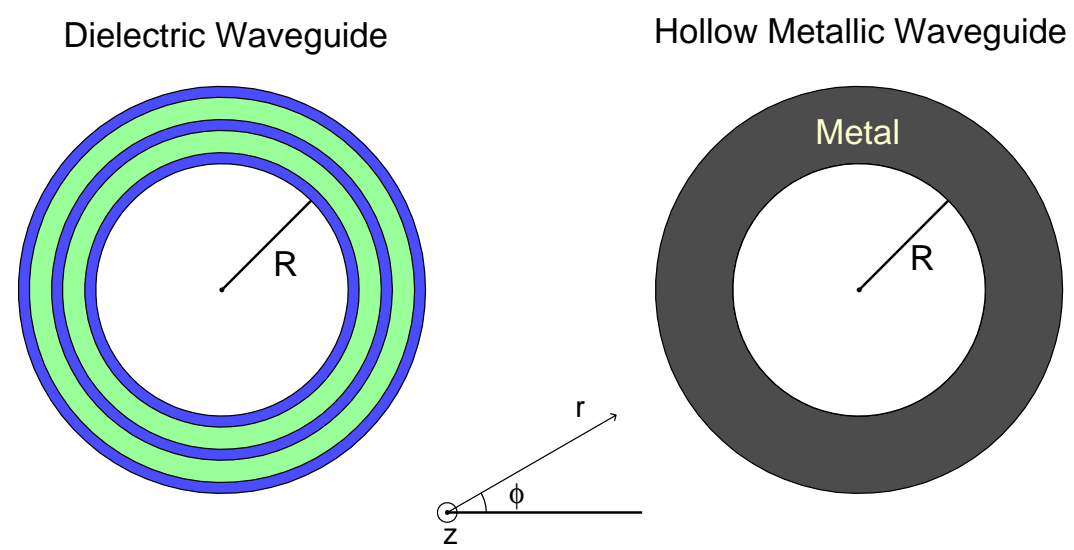

Figure 1. (a) Hollow dielectric waveguide of radius $R$. Light is confined in the hollow core by a multilayer dielectric mirror made of alternating layers with high and low indices of refraction. (b) Hollow metallic waveguide of radius $R$. Light is confined in the hollow core by a metallic cylinder.

One of the hallmarks of photonic crystals is their flexibility, since their optical properties are subject to deliberate structural and materials choices. OmniGuide fibers also embody this freedom, permitting a wide variety of layer-thickness designs to enhance or inhibit specific characteristics (e.g. to tailor dispersion parameters), which we do not explore here. Moreover, due to the suppression of cladding properties, a much broader range of materials is available for use than would normally be practical in low-loss optical fibers. In this paper, we describe methods for understanding and designing OmniGuide fiber properties, and especially focus on general scaling laws, phenomena, and design tradeoffs that apply in such systems. For a particular example system, we arbitrarily select an index contrast of 4.6 to 1.6 in the cladding, as has been used in several previous publications $[7,10,11,13-15]$. Our scaling laws, analytical techniques, and qualititative results, however, hold true for a wide range of alternative parameters, as was further analyzed in [15].

\section{HOLLOW DIELECTRIC VS. METALLIC WAVEGUIDES}

In this section, we begin by introducing the basic structure and principles of operation for OmniGuide fibers, and develop an intuition for their behavior by an analogy with hollow metallic waveguides [19, 20]. One could also compare with other prior work, including the hybrid system of a metallic waveguide whose inner surface is coated with a multilayer Bragg mirror [21,22], as well as several other hollow waveguiding systems [23], but the pure metallic guide provides the simplest foundation for understanding. In the left panel of Fig. 1, we depict a schematic of an OmniGuide fiber forming a hollow dielectric waveguide. A hollow core (index of refraction unity) of radius $R$ is surrounded by a multilayer cladding that consists of alternating layers having high and low indices of refraction. As discussed above, we choose indices of refraction 4.6 and 1.6, with thicknesses here of $0.33 a$ and $0.67 a$, where $a$ is the thickness of one high/low bilayer (we select different thicknesses in Sec. 2.2). Once a mode frequency $\nu$ is computed in units of $c / a$, the physical value of $a$ is determined via $a=\lambda \nu$ for some desired operational wavelengh $\lambda[1]$. The radius $R$ of the waveguide will vary in the differing examples presented in this paper, from a minimum of $2 a$ to a maximum of $30 a$. In the right panel of Fig. 1, we show a hollow metallic waveguide. The core is the same as that of the hollow dielectric waveguide, but a metal cylinder now replaces the multilayer cladding.

In the metallic case, light is confined in the core by the impenetrability of a near-perfect metal (nonexistent at optical frequencies) - such confined modes for $R=2 a$ are depicted in the right panel of Fig. 2. Dispersions relations like this one depict two conserved quantities: the axial wavenumber $\beta$ and the frequency $\omega$. In cylindrical waveguides, modes can also be labeled by their "angular momentum" integer $m$. For waveguides that lie along the $z$ axis, the $(z, t, \varphi)$ dependence of the modes is then given by: $e^{i(\beta z-\omega t+m \varphi)}$. In a hollow metal 

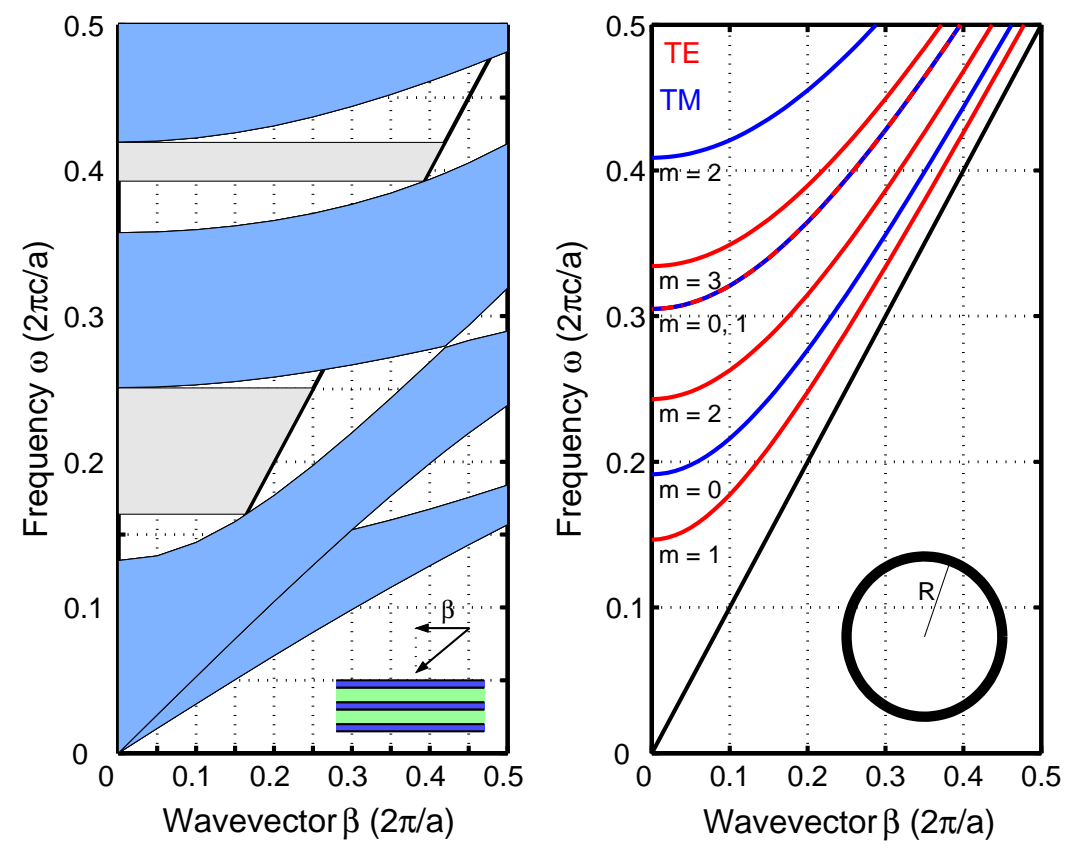

Figure 2. (Left) Projected band structure associated with the planar dielectric mirror. The dark shaded regions correspond to $(\beta, \omega)$ pairs for which light can propagate within the mirror. White and light gray regions correspond to situations where light cannot propagate within the mirror. The thick black line represents the light line $(\omega=c \beta)$. Shown in gray are the two omnidirectional frequency ranges of the mirror. (Right) Dispersion relations $\omega(\beta)$ of the lowest 7 modes supported by a hollow metallic waveguide of radius $R=2 a$ are plotted. The modes have angular dependence $e^{i m \varphi}$. Note the degeneracy of the $\mathrm{TE}_{01}$ and the $\mathrm{TM}_{11}$ modes.

tube, the eigenmodes are purely polarized as TM $\left(H_{z}=0\right)$ or TE $\left(E_{z}=0\right)$, and the $\ell$-th mode of a given $m$ is labeled $\mathrm{T} X_{m \ell}$.

In the dielectric case, light is confined by the one-dimensional photonic band gap of the multi-layer cladding, which is easy to analyze in the limit as the cladding becomes planar. The one-dimensional gaps of the planar dielectric mirror structure as a function of $\beta$ (the surface-parallel wavevector component) are depicted in the left panel of Fig. 2. In these gap regions, we expect the mirrors to behave similarly to a metal, and confine modes strongly analogous to those of the metallic waveguide; this is verified below. Because every eigenmode has a finite, conserved $m$, the effective wavevector $k_{\varphi}=m / r$ in the $\hat{\varphi}$ direction goes to zero for $r \rightarrow \infty$. Without this fact, there would be no band gaps in Fig. 2, since nonzero $k_{\varphi} \perp \beta$ would have to be projected onto the Bragg band diagram. Also shown in this figure, as gray regions, are the ranges of omnidirectional reflection: the frequencies at which any incident wave from air will be reflected by the planar mirrors (and vice versa). Omnidirectional reflection per se is not strictly necessary for guidance in these fibers, but its presence is strongly correlated with the regimes of large, polarization-independent gaps along the light line that are important for many of the properties studied in this paper.

Bragg mirrors have different band-gaps for "TE" and "TM" polarizations, referring to fields purely parallel to the interface and fields with a normal component, respectively. (Both polarizations are shown in Fig. 2.) This corresponds to the waveguide TE and TM labels only for $m=0$; all $m \neq 0$ modes have some nonzero $E_{r}$ component. 


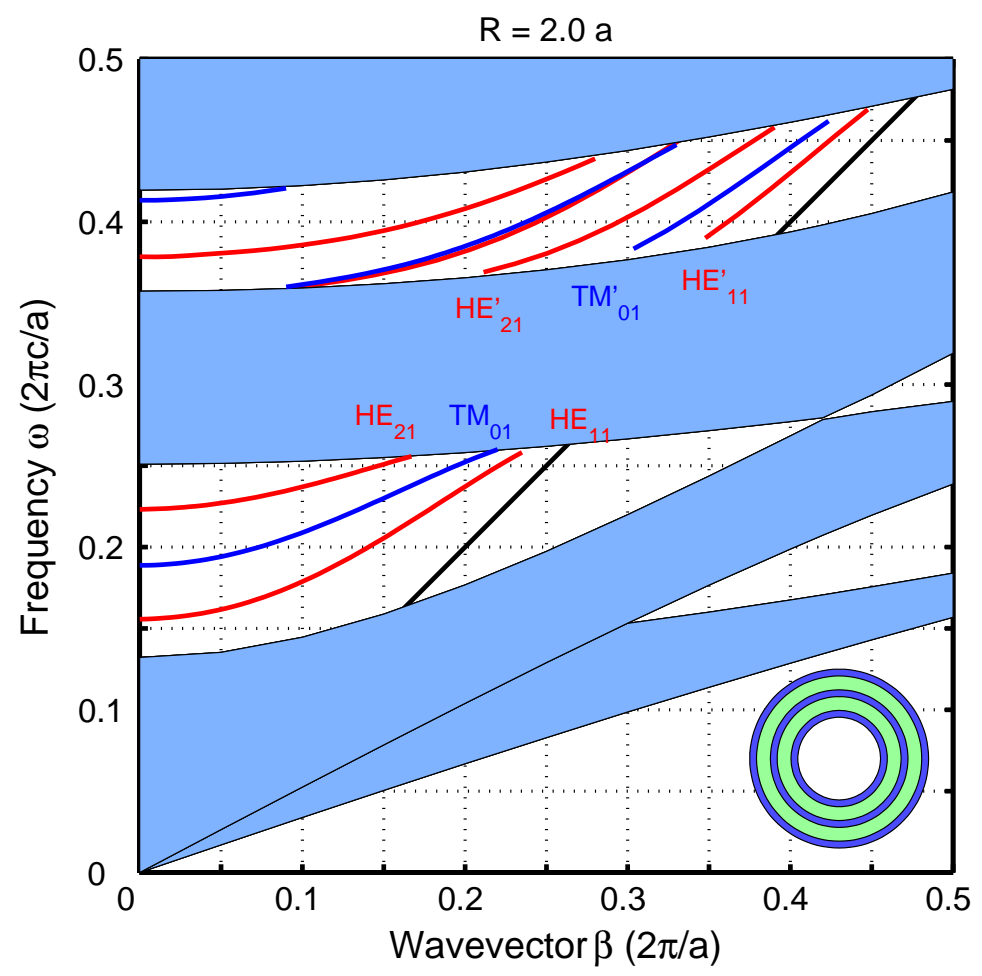

Figure 3. Guided modes supported by a hollow OmniGuide fiber of radius $R=2 a$ : TE/HE modes and TM/EH modes. In black is the light line $(\omega=c \beta)$, and the dark shaded regions represent the continuum of modes that propagate within the multilayer cladding. Only the first three modes in each band gap are labeled.

\subsection{The modes in an OmniGuide fiber}

The modes supported by any cylindrical waveguide, including metallic waveguides, OmniGuide fibers, and traditional silica fibers, can be computed by the transfer-matrix method of [3]. Here, the longitudinal fields $\left(E_{z}\right.$ and $\left.H_{z}\right)$ of a given $(m, \omega, \beta)$ in an annular region of index $n_{j}$ are expanded in Bessel functions $J_{m}\left(k_{j} r\right)$ and $Y_{m}\left(k_{j} r\right)$, with $k_{j} \equiv \sqrt{n_{j}^{2} \omega^{2} / c^{2}-\beta^{2}}$. At each interface, the coefficients are related by a $4 \times 4$ transfer matrix that matches boundary conditions. The product of all these transfer matrices yields a single matrix relating the fields in the core to those in the outermost cladding. Then, by application of appropriate boundary conditions, the $\beta_{n}$ wavevectors of the various modes can be found; this is discussed in more detail by Sec. 4 below.

Here, we are primarily interested in the modes that lie within the band gap of the one-dimensional Bragg mirrors. Such modes must decay exponentially with $r$ in the cladding, and therefore are truly guided modes in the limit of infinitely many cladding layers (the case of finite layers is considered in Sec. 4). Most of these modes lie above the $\omega=c \beta$ light line, and thus propagate within the hollow core in much the same way as the modes of a metallic waveguide. It is also possible, however, for modes to lie beneath the light line and yet inside the band gap, in which case they are surface states localized around the core/cladding interface- we discuss this possibility further below.

In Fig. 3, we show the computed guided modes of the OmniGuide fiber with core-radius $R=2 a$ and the abovementioned planar-mirror parameters. These modes are at nearly the same frequencies as the metallic waveguide modes of Fig. 2, with the one-dimensional bandgaps simply superimposed. In the dielectric waveguide, the modes are only purely TE and TM for $m=0$, but for $m \neq 0$ they are strongly TE-like or TM-like, and are 


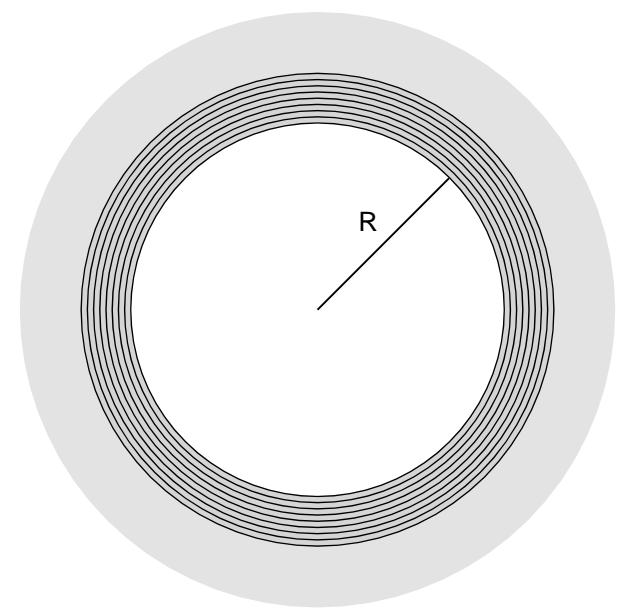

Figure 4. An OmniGuide fiber with core radius $R=30 a$, the parameters that we employ in the remainder of this paper. The omnidirectional mirror here comprises 17 layers, starting with a high-index layer, with indices 4.6/1.6 and thicknesses $0.22 a / 0.78 a$, respectively. (The omnidirectional mirror is surrounded by some coating for mechanical support; this layer is not shown to scale.) We choose $a=0.434 \mu \mathrm{m}$, so that the lowest dissipation losses occur roughly at $\lambda=1.55 \mu \mathrm{m}$.

called $\mathrm{HE}$ and $\mathrm{EH}$, respectively. (When a mode enters the second gap, we add a prime superscript.) Moreover, because these modes are so strongly confined within the core, the field patterns must also be nearly identical. We consider this analogy in greater detail in a later publication [16].

\subsection{A Large-core OmniGuide fiber}

The above calculations yielded the modes of an OmniGuide fiber for a radius $R=2 a$. This small radius has the advantage of supporting only a few modes, which are easy to plot and understand in their entirety, and even has a single-mode frequency range. The analogy with metallic waveguides, however, tells us that this may not be the most desirable regime for fiber operation. In this section, we motivate the use of larger, ostensibly multi-mode cores for OmniGuide fibers, and describe the fiber parameters that we will use for subsequent computations in this paper.

In metallic waveguides, the lowest-loss mode is $\mathrm{TE}_{01}$, and its ohmic losses decrease as $1 / R^{3}[19,20]$. Moreover, the differential losses between $\mathrm{TE}_{01}$ and other modes create a modal-filtering effect that allows these waveguides to operate in an effectively single-mode fashion. On the other hand, for large core radii (high frequencies), losses become dominated by scattering into other closely-spaced modes, especially into the degenerate $\mathrm{TM}_{11} \mathrm{mode}$ via bends, and it was found that the optimal radius was in the range of $4 \lambda-11 \lambda[20]$.

Similar results hold for OmniGuide fibers: the lowest-loss mode is $\mathrm{TE}_{01}$, and many of its losses fall off as $1 / R^{3}$ (for much the same reasons as in metallic waveguides). Like the metallic waveguides, and unlike silica fibers with their small material contrasts, we demonstrate a strong modal-filtering effect based on the degree of confinement in the core. Also as before, inter-modal scattering worsens with increasing $R$. (Bending is less of a problem than in metallic waveguides, however, since the dielectric cladding breaks the $\mathrm{TE}_{01} / \mathrm{TM}_{11}$ degeneracy [15].) Thus, for the example OmniGuide fiber that we use in the remainder of this paper, we choose a core radius of $R=30 a$, as depicted in Fig. 4. The point of lowest $\mathrm{TE}_{01}$ dissipation losses that we compute in Sec. 6.1 then lies at a frequency of $\omega \cong 0.28 \cdot 2 \pi c / a$, so if we make this correspond to the standard $\lambda=1.55 \mu \mathrm{m}$ of telecommunications, we have $a=0.434 \mu \mathrm{m}$. Equivalently, $R=13.02 \mu \mathrm{m}=8.4 \lambda$, in the favorable range for the metallic-waveguide analogy. Throughout this paper, however, we will emphasize scaling laws with $R$ in order to highlight the effects of differing core sizes.

In order to choose the layer thickness, we employed an approximate quarter-wave condition. It is well-known that, for normal incidence, a maximum band gap is obtained for a "quarter-wave" stack in which each layer has 

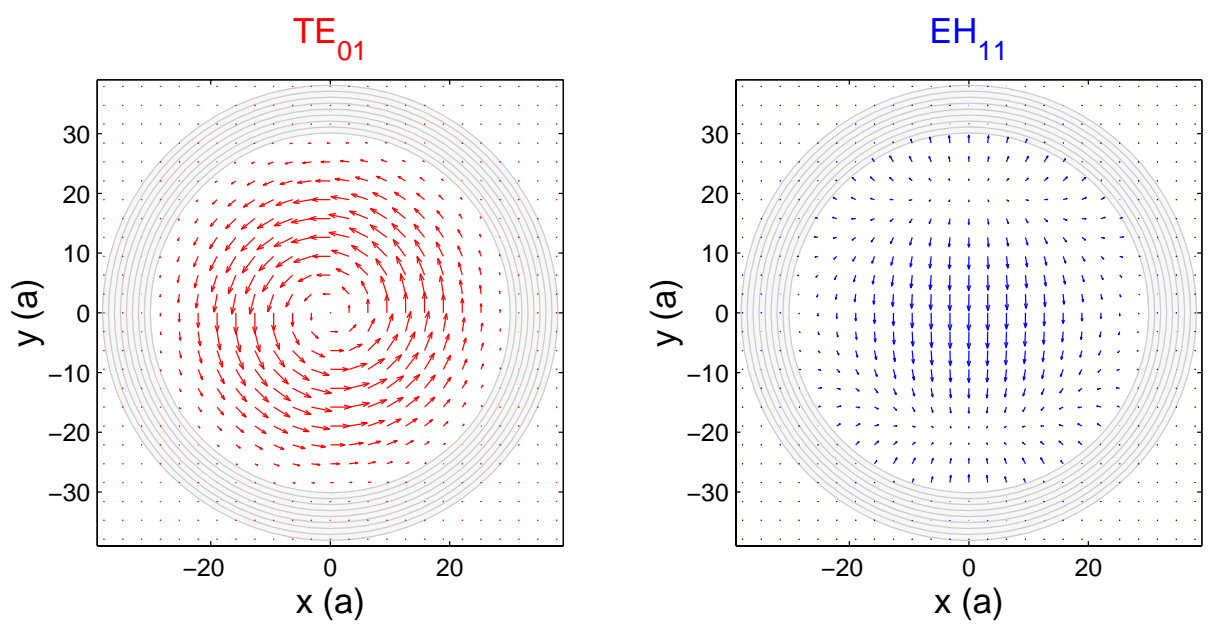

Figure 5. Transverse electric-field distributions in the OmniGuide fiber of Fig. 4 for the $\mathrm{TE}_{01}$ mode (left) and the $\mathrm{EH}_{11}$ mode (right), which have $\beta=0.27926 \cdot 2 \pi / a$ and $\beta=0.27955 \cdot 2 \pi / a$, respectively, at $\omega=0.28 \cdot 2 \pi c / a$.

equal optical thickness $\lambda / 4: d_{\text {hi }} / d_{\text {lo }}=n_{\text {lo }} / n_{\text {hi }}[24]$. Normal incidence, however, corresponds to $\beta=0$, whereas the modes of interest in the OmniGuide fiber lie almost on the $\beta=\omega / c$ light line (in the limit of $R \rightarrow \infty$, the lowest-order modes are essentially plane waves propagating along $\hat{z}$ ). Thus, we employ layer thicknesses determined by the quarter-wave condition along the light line of air (similarly applied in [21]):

$$
\frac{d_{\mathrm{hi}}}{d_{\mathrm{lo}}}=\frac{\sqrt{n_{\mathrm{lo}}^{2}-1}}{\sqrt{n_{\mathrm{hi}}^{2}-1}}
$$

which yields $d_{\mathrm{hi}} \cong 0.2176 a$ and $d_{\mathrm{lo}} \cong 0.7824 a$ in this case. (Ref. [3] suggests an alternate method that optimizes the structure for confinement of a given mode, but this yields essentially the same thicknesses as Eq. (1) for large $R$.)

As in the $R=2 a$ case, the guided-modes of this $R=30 a$ OmniGuide fiber can be labeled by analogy to the modes of an equal-radius metallic waveguide. Two such modes in the OmniGuide fiber, the lowest-loss $\mathrm{TE}_{01}$ and the linearly-polarized $\mathrm{EH}_{11}$ (analogous to the $\mathrm{TM}_{11}$ mode in a metallic guide) are depicted in Fig. 5. The $\mathrm{TE}_{01}$ mode is circularly symmetric and "azimuthally" polarized $(\vec{E} \| \hat{\varphi})$ - thus, unlike the doubly-degenerate $\mathrm{EH}_{11}$ mode (two orthogonal polarizations), $\mathrm{TE}_{01}$ cannot be split into two modes of differing velocities by fiber imperfections, and is therefore immune to polarization-mode dispersion (PMD). (See also Sec. 8.)

\section{SCALING LAWS WITH CORE SIZE}

Because of the strong reflectivity of the dielectric mirrors, many of the mode properties are determined largely by the geometric size $R$ of the core, within which the modes are confined. Throughout this paper, we thereby derive scaling relations for the different quantities computed, and in this section we lay the groundwork for those derivations by presenting basic scalings of the fields and modes. These scaling relations are largely independent of details such as the precise index contrast that is used, so long as it is sufficiently large for the metallic analogy to hold, and will provide a broad understanding of the advantages and tradeoffs of OmniGuide fiber structures. Later in the paper, we exhibit data to explicitly verify scaling relations derived from those in this section.

In particular, we will focus on the suppression of cladding phenomena for the $\mathrm{TE}_{0 \ell}$ (especially $\mathrm{TE}_{01}$ ) modes of the fiber. The critical property of $\mathrm{TE}_{0 \ell}$ modes is that, by analogy with a hollow metallic waveguide, they have a node in their electric field $\left(E_{\varphi}\right)$ near $r=R[19,20]$. It then follows that the amplitude of the electric 
field in the cladding is proportional to the slope of $E_{\varphi}$ at $R$. The form of $E_{\varphi}$ in the core, however, is simply the Bessel function $J_{1}(\xi r / R)$, where $\xi(\omega)$ is roughly the $\ell$-th zero of $J_{1}$. The slope at $R$ is then $\left(J_{0}(\xi)-J_{2}(\xi)\right) \cdot \xi / 2 R$. Moreover, for our quarter-wave stack, $E_{\varphi}$ peaks near each of the $n_{\mathrm{hi}} \rightarrow n_{\text {lo }}$ interfaces [3]. Thus, not including any normalization of the $J_{1}$ amplitude $\left(\right.$ i.e. $E_{\varphi} \sim 1$ ), we find that the unnormalized $E_{\varphi}$ in the cladding scales as $d_{\mathrm{hi}} / R$.

In addition, for most computations (such as the perturbation theory described in the appendix), one must normalize the power of the field: this means dividing $\vec{E}$ by an additional factor proportional to $\sqrt{\text { mode area }} \sim R$, and so:

$$
\text { normalized } \mathrm{TE}_{0 \ell} \text { cladding } \vec{E} \sim \frac{1}{R^{2}} \text {. }
$$

Moreover, the area of the field in the cladding is the perimeter $\sim R$ times some constant (penetration depth) that depends on the size of the band gap; combined with Eq. (2), we therefore find:

$$
\text { fraction of } \int|\vec{E}|^{2} \text { in cladding for } \mathrm{TE}_{0 \ell} \sim \frac{1}{R^{3}},
$$

and from this we derive many other scaling relations. In contrast, for TM or mixed-polarization modes with an $E_{r}$ component, the unnormalized field amplitude in the cladding remains roughly constant with changing $R$-their fractional $|\vec{E}|^{2}$ in the cladding then scales as only $1 / R$, so the cladding has a much greater effect on them.

\section{LEAKY MODES AND RADIATION LOSS}

In the preceding discussion, we have neglected a point that may seem important: in reality, there will be only a finite number of cladding layers in the omnidirectional mirror. Because of this, and the fact that the modes of interest lie above the light line of the outermost region, the field power will slowly leak out in a process akin to quantum-mechanical "tunneling." This radiation loss, however, decreases exponentially with the number of cladding layers, and we quantify its smallness explicitly below. As has been observed elsewhere [11, 13, 15], we show that only a small number of layers is required to achieve leakage rates well below $0.1 \mathrm{~dB} / \mathrm{km}$. Moreover, the radiation leakage strongly differs between modes, inducing a modal-filtering effect $[3,6]$ that allows a large-core OmniGuide fiber to operate in an effectively single-mode fashion.

In the limit of infinitely many cladding layers, the modes in the OmniGuide core are true confined modes, thanks to the band gap, with discrete real eigenvalues $\beta_{n}$ (c.f. the appendix). For finitely many layers, modes are no longer truly confined (above the outermost light line), leading to a continuum of $\beta$ values with infinitely-extended eigenstates [3]. The former confined modes become leaky resonances: superpositions of real$\beta$ eigenmodes centered on $\beta_{n}$ with a width $\Delta \beta$ proportional to the radiative decay rate $\alpha_{n}$. Such resonances can be studied directly via the physical real- $\beta$ continuum, but a powerful alternative method is the leaky-mode technique, which employs a conceptual analytic continuation from $\beta$ to $\widetilde{\beta}$ in the complex plane to satisfy a boundary condition of zero incoming flux [25]. The power decay rate $\alpha_{n}$ is then given by $2 \Im\left[\widetilde{\beta}_{n}\right]$, where $\Im$ denotes the imaginary part; we have also verified the correctness of this decay rate against explicit real- $\beta$ superpositions as well as by beam-propagation methods (BPM) [26].

For an OmniGuide fiber, the leaky-mode method is as follows. The transfer-matrix formulation allows one to compute $2 \times 2$ matrices $M_{m}^{ \pm}(\omega, \beta)$ that connect the TE and TM amplitudes at the origin to the amplitudes of the outgoing $(+)$ or incoming (-) TE and TM cylindrical waves (Hankel functions) in the outermost region, as a function of $\omega$ and $\beta$ for a given angular-momentum index $m$. (For $m=0$, the two polarizations decouple [3].) For a leaky mode, we wish to impose the boundary condition of zero incoming waves, so that there are no sources at $\infty$; such a solution exists whenever there is a zero eigenvalue of $M_{m}^{-}$. Therefore, we use the determinant:

$$
f_{m}(\omega, \widetilde{\beta}) \equiv \operatorname{det}\left[\mathrm{M}_{m}^{-}(\omega, \widetilde{\beta})\right]
$$

so that the leaky mode is defined by $f_{m}\left(\omega, \widetilde{\beta}_{n}\right)=0$. Once such a $\widetilde{\beta}_{n}$ is found for a given $\omega$, the corresponding eigenvector of $M_{m}^{-}\left(\omega, \widetilde{\beta}_{n}\right)$ yields the required mixed-polarization amplitudes. With finitely many layers, the only 


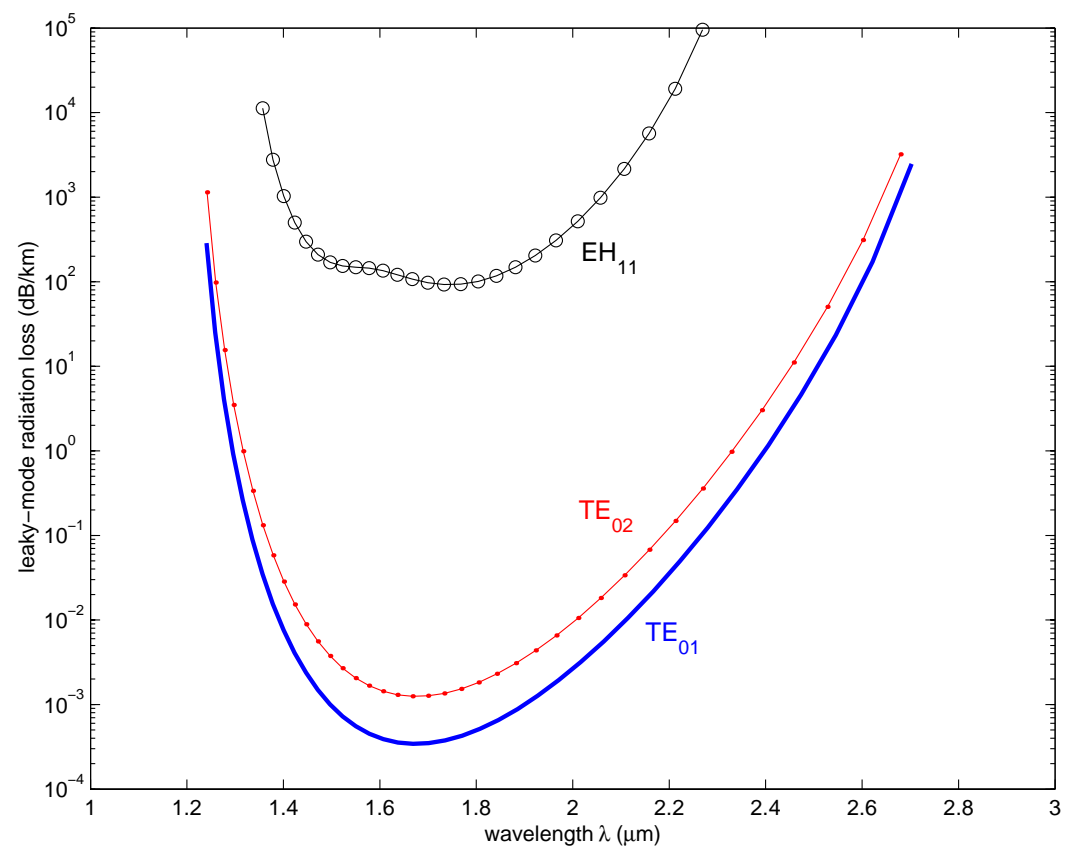

Figure 6. Radiation leakage through a finite number (17) of cladding layers in the OmniGuide fiber of Fig. 4. The lowest-loss mode is $\mathrm{TE}_{01}$ (solid) and the next-lowest is $\mathrm{TE}_{02}$ (dots), while the linearly-polarized $\mathrm{EH}_{11}$ mode (circles) typifies the higher losses for mixed-polarization modes due to the smaller TM band gap.

real roots of $f_{m}$ lie below the light line of the outermost region; above this light line, the incoming and outgoing flux are equal for real $\beta[3]$, corresponding to steady-state standing-wave patterns. The small imaginary part of $\widetilde{\beta}_{n}$ above the light line yields the power decay rate $\alpha_{n}=2 \Im\left[\widetilde{\beta}_{n}\right]$. A similar leaky-mode method was previously used for Bragg fibers, albeit with only a first-order Taylor-expansion approximation for $\Im\left[\widetilde{\beta}_{n}\right][5]$.

For all modes, the radiative decay $\alpha$ decreases exponentially with increasing numbers of cladding layers, thanks to the exponential decay of fields in the Bragg band gap, eventually to the point where other losses (e.g. absorption) dominate. At $\lambda=1.55 \mu \mathrm{m}$ for this structure, the TE/TM losses decrease by a factor of $\sim 10 / 5$ per cladding bilayer, respectively. Because of the smaller TM band gap, the losses of mixed-polarization $(m \neq 0)$ modes are eventually dominated by their less-confined TM components. In Fig. 6, we display the computed radiation leakage rates $\alpha$ for the lowest-loss $\mathrm{TE}_{01}$ mode, the next-lowest loss $\mathrm{TE}_{02}$ mode, and the linearly-polarized $\mathrm{EH}_{11}$ mode to typify mixed-polarization modes. Like the absorption discussed later, these differential losses create a mode-filtering effect that allows the $\mathrm{TE}_{01}$ mode to operate as effectively single-mode, even for large-core OmniGuide fibers, a fact that was also noted in [3,6]. A similar principle was employed in hollow metallic waveguides $[19,20]$ to select the $\mathrm{TE}_{01}$ mode. Note that, as in metallic waveguides, $\mathrm{TE}_{02}$ is not necessarily the mode of greatest concern - it is more important to consider modes that couple strongly to $\mathrm{TE}_{01}$ via perturbations, such as those discussed [15]. From Fig. 6, it is seen that with only 17 cladding layers the $\mathrm{TE}_{01}$ mode has leakage rates well under $0.01 \mathrm{~dB} / \mathrm{km}$, and even $\mathrm{EH}_{11}$ has decay lengths of meters, corresponding to $\Im\left[\widetilde{\beta}_{n}\right] / \Re\left[\widetilde{\beta}_{n}\right]$ of $\sim 10^{-13}$ and $\sim 10^{-9}$, respectively. Thanks to these low losses, the modes can be treated as truly bound for most analyses (e.g. dispersion relations and perturbation theory), with the leakage rates at most included as an independent loss term.

The radiation losses are proportional to the field amplitude $|\vec{E}|^{2}$ in the cladding, which goes like $1 / R^{4}$ for $\mathrm{TE}_{0 \ell}$ from Eq. (2), multiplied by the surface area (which scales as $R$ ). Thus,

$\mathrm{TE}_{0 \ell}$ radiation leakage $\alpha \sim 1 / R^{3}$, 


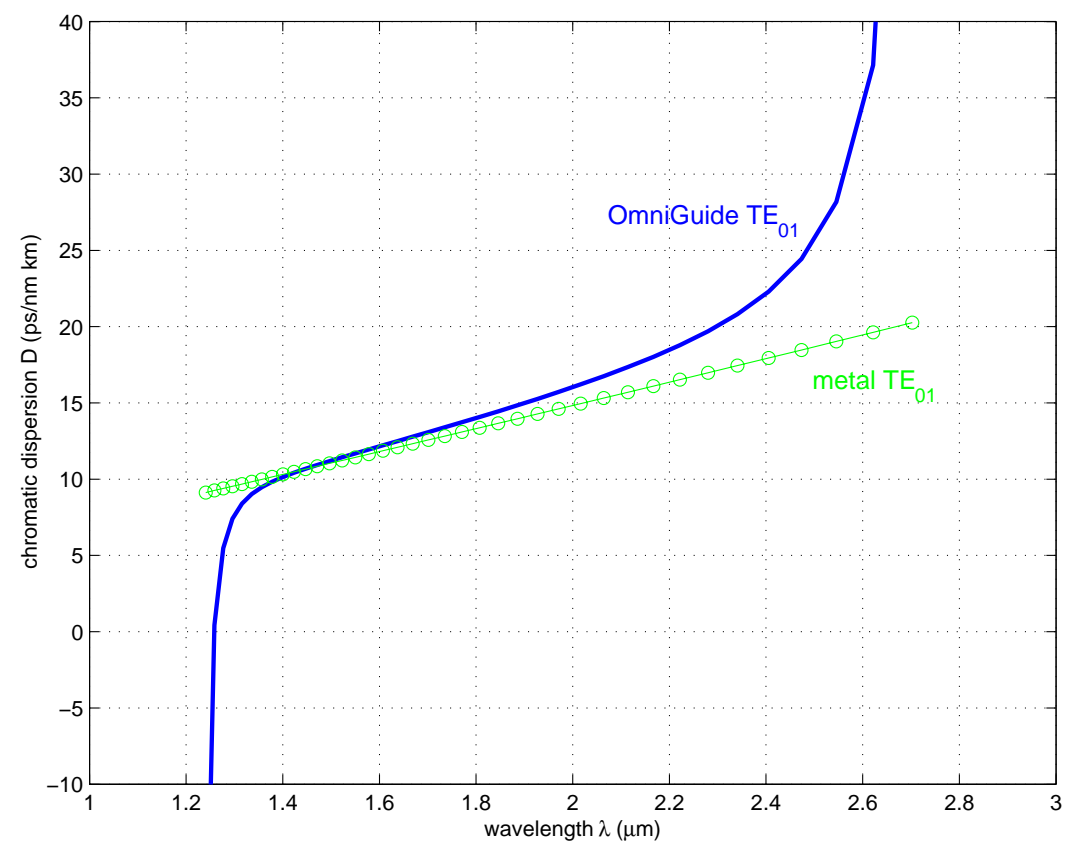

Figure 7. Group-velocity (chromatic) dispersion of the $\mathrm{TE}_{01}$ mode in both the OmniGuide fiber of Fig. 4 (solid) and a hollow metallic waveguide with the same core radius (circles).

the same as the scaling of $\mathrm{TE}_{0 \ell}$ ohmic losses in a hollow metallic waveguide $[19,20]$. In contrast, because of their lack of a node near the boundary, TM and mixed-polarization radiation losses scale only as $1 / R$.

\section{GROUP-VELOCITY DISPERSION}

Given a dispersion relation $\beta(\omega)$, one important quantity is the group-velocity dispersion $D$ (the rate at which pulses spread), canonically defined as [18]:

$$
D \equiv-\frac{\omega^{2}}{2 \pi c} \frac{d^{2} \beta}{d \omega^{2}}
$$

in units of $\mathrm{ps} /(\mathrm{nm} \cdot \mathrm{km})$ : the pulse-spreading $(\mathrm{ps})$ per $\mathrm{km}$ of propagation per $\mathrm{nm}$ of $\Delta \lambda$. This can be computed exactly from the function $f$ of Eq. (4), which defines the dispersion relation implicitly by $f(\omega, \beta)=0$. The group velocity is thus given by $v \equiv d \omega / d \beta=-f_{\beta} / f_{\omega}$, where the subscripts denote partial differentiation, and one thereby finds that $d^{2} \beta / d \omega^{2}=d(1 / v) / d \omega=2 f_{\omega} f_{\omega \beta} / f_{\beta}^{2}-f_{\omega \omega} / f_{\beta}-f_{\omega}^{2} f_{\beta \beta} / f_{\beta}^{3}$. We evaluate all of these derivatives analytically by differentiating the transfer matrices. We also considered material dispersion $(n$ varying with $\omega)$ by the same methods, but we found that this has a negligible effect (due to the small field penetration into the cladding). For example, assuming that the cladding has the same $d n / d \omega$ and $d^{2} n / d \omega^{2}$ as silica at $1.55 \mu \mathrm{m}$, the contribution of material dispersion is less than $0.1 \mathrm{ps} /(\mathrm{nm} \cdot \mathrm{km})$ over most of the bandwidth. What remains is the waveguide dispersion, which stems from the geometry of the core as well as the variable penetrability of the cladding, Goos-Hänchen shifts [24], etc., all of which are taken into account by differentiating $f$. The resulting dispersion as a function of wavelength is plotted in Fig. 7 for the $\mathrm{TE}_{01}$ mode of the $R=30 a$ OmniGuide fiber.

For comparison, we also plot the dispersion of the $\mathrm{TE}_{01}$ mode in an equal-radius hollow metallic waveguide, given by: $D_{\text {metal }}=-\omega^{2} \xi^{2} /\left(2 \pi c^{3} \beta^{3} R^{2}\right)$, where $\beta=\sqrt{\omega^{2} / c^{2}+\xi^{2} / R^{2}}$ and $\xi=3.8317 \cdots$ is the first non-zero root of the $J_{1}$ Bessel function. Except near the edges of the band gap, the dispersion is very similar to that of the metallic waveguide - that is, $D$ is dominated simply by the core shape - and $D \cong 12 \mathrm{ps} / \mathrm{nm} \cdot \mathrm{km}$ at $\lambda=1.55 \mu \mathrm{m}$. From the metallic dispersion relation, we can also conclude that $D \sim 1 / R^{2}$. 
As we discuss in the next section, the practical implications of dispersion in an OmniGuide fiber are quite different than in ordinary fibers, due to the absence of nonlinear effects. Because dispersion no longer interacts with nonlinearities, it can in principle be completely compensated after any distance of propagation, allowing one to put all dispersion compensation at the end of a fiber link, as well as to tolerate higher dispersions. Conversely, operating at or near a point of zero dispersion will no longer exacerbate four-wave mixing noise.

Another important consideration is the relative dispersion slope (RDS), as measured by $(d D / d \lambda) / D$; this quantity must ideally be matched in any dispersion-compensation system. For the OmniGuide fiber above, the RDS is around $0.0007 \mathrm{~nm}^{-1}$. This is $15-30$ times smaller than the RDS of contemporary TrueWave-RS $\left(0.010 \mathrm{~nm}^{-1}\right)$ and LEAF $\left(0.021 \mathrm{~nm}^{-1}\right)$ fibers, and smaller slopes are typically easier to achieve in dispersioncompensating fibers [27].

\section{SUPPRESSION OF ABSORPTION AND NONLINEARITIES}

In this section, we compute the effect of absorption losses and nonlinearities in the cladding materials of an OmniGuide fiber. We show that these effects are strongly suppressed for the $\mathrm{TE}_{01}$ mode, allowing highly lossy and nonlinear materials to be employed - greatly broadening one's choices for high-index materials. Moreover, we will see that there is the potential of greatly surpassing even the properties of silica fibers.

Absorption and nonlinearites correspond to tiny shifts $\Delta \varepsilon$ in the dielectric constant of the materials, and can therefore be treated by perturbation theory, as described in detail by the appendix. This common technique allows one to compute the shift $\Delta \beta$ due to a small perturbation, using only the unperturbed modes (computed earlier via transfer matrices). We employ erturbation-theory expressions for the absorption loss and nonlinearities that have been previously derived, and which we have also derived in [15] via explicit analogy with quantum mechanics. In both cases, the relevant quantity that we compute is the lowest-order shift in $\beta$ due to a small $\Delta \varepsilon$ in the structure.

\subsection{Absorption Loss}

For absorption losses (also possibly including Rayleigh scattering), $\Delta \varepsilon$ is a small imaginary part added to $\varepsilon$, representing the (material-dependent) dissipation rate. The resulting (purely imaginary) $\Delta \beta$ indicates the decay rate of the mode, as derived in [15,28-33].

Here, we calculate the losses of the $\mathrm{TE}_{01}$ mode in our example OmniGuide fiber by assuming that the core is lossless, and that both the high and low-index cladding materials have the same power dissipation rate $\alpha_{0}$ (for propagation in bulk). Furthermore, we divide the computed dissipation rate $\alpha$ by $\alpha_{0}$, yielding the dimensionless, material-loss independent absorption suppression coefficient of the mode. This is done for each frequency $\omega$ across the band gap, yielding the plot in Fig. 8. Thus, the cladding losses are suppressed by more than four orders of magnitude over most of the bandwidth, a result that will be better understood from the scaling-law arguments in Sec. 6.3. For comparison, we also show the next-lowest loss $\mathrm{TE}_{02}$ mode, as well as the linearly-polarized $\mathrm{EH}_{11}$ (exemplifying the larger cladding penetration for modes with TM components). As was mentioned earlier, such a loss differential creates a mode-filtering effect that should allow single-mode-like operation. (Surface states and guided modes outside the band gap, i.e. propagating within the cladding region, will experience essentially the attenuation of the bulk cladding materials and will be even more strongly filtered out.) Moreover, even if the cladding has losses a thousand times greater than silica's $\sim 0.2 \mathrm{~dB} / \mathrm{km}$, the $\mathrm{TE}_{01}$ losses can be lower than the losses of silica fibers.

\subsection{Nonlinearities}

Another important problem in optical fibers is that of Kerr nonlinearities. Here, the index $n$ of the material varies as a function of electric-field strength: $n^{\prime} \equiv n+n_{2}|\vec{E}|^{2}$, where $n_{2}=\frac{3}{8 n} \chi^{(3)}$ is the (small) "nonlinear index coefficient" of the material(s). ${ }^{\dagger}$

\footnotetext{
${ }^{\dagger}$ Often, one instead uses $n^{\prime}=n+n_{2} I$, where $I \sim n|\vec{E}|^{2}$ is the intensity of light; our analysis remains the same except for the extra factor of $n$.
} 


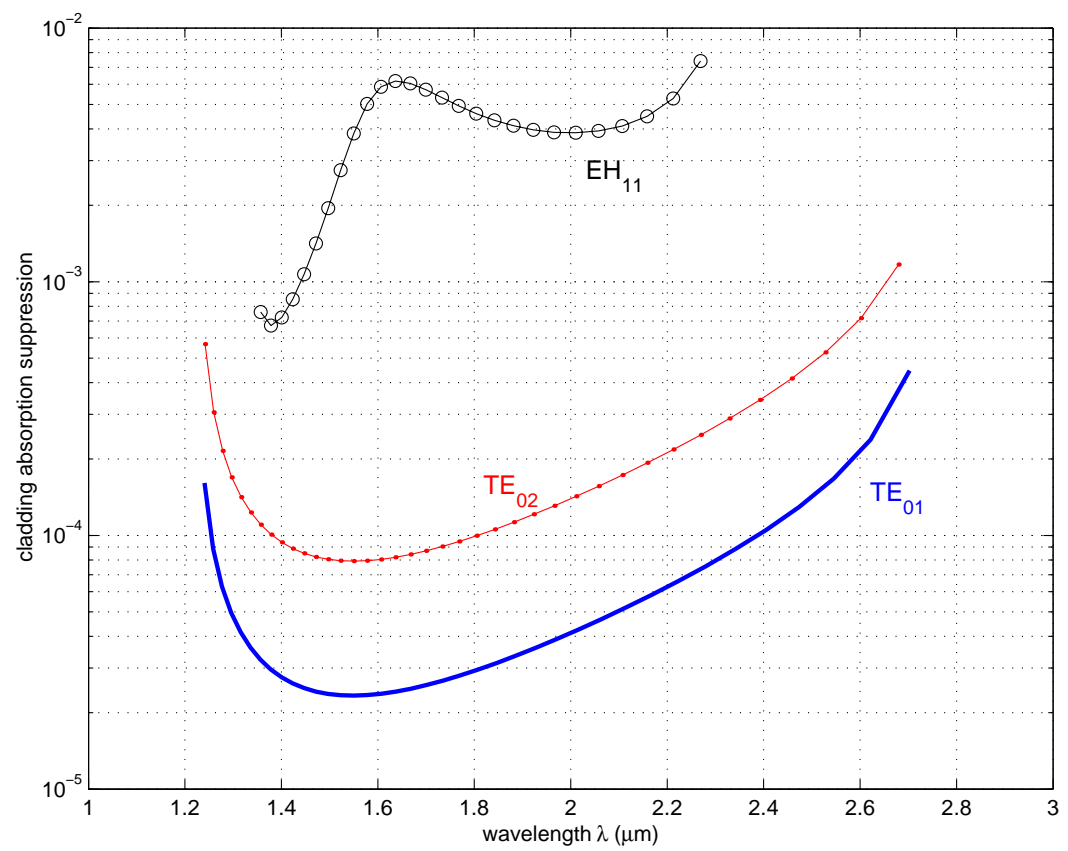

Figure 8. Absorption losses due to the cladding materials the OmniGuide fiber (with core radius 30a), as a fraction of the bulk cladding losses. The lowest-loss mode is $\mathrm{TE}_{01}$ (solid) and the next-lowest is $\mathrm{TE}_{02}$ (dots), while the linearly-polarized $\mathrm{EH}_{11}$ mode (circles) typifies the higher losses for mixed-polarization modes due to the smaller TM band gap.

Thus, to first order in $n_{2}$, one has:

$$
\Delta \varepsilon=2 n n_{2}|\vec{E}|^{2}
$$

Kerr nonlinearities cause several problems in fiber systems: self/cross-phase modulation (SPM/XPM), where the energy at one frequency shifts the $\beta$ at the same/another frequency; and also four-wave mixing (FWM), in which energy at one frequency leaks into another frequency [18]. SPM and XPM interact with dispersion to limit dispersion-compensation schemes, and FWM causes noise/crosstalk between channels. Our concern here is not to compute these effects per se, but rather to define the limits in which they may be neglected.

The strength of nonlinearities in a fiber is given by a nonlinear lengthscale $L_{\mathrm{NL}}$, defined as the inverse of the SPM phase shift $\Delta \beta$; this is the lengthscale at which SPM and XPM become significant, and also appears as a scaling coefficient in the FWM noise [18]. As is seen below, $L_{\mathrm{NL}}$ is inversely proportional to the mode power $P$ (to first order), so it is conventional to instead define the nonlinear strength $\gamma \equiv 1 /\left(P L_{\mathrm{NL}}\right)=\Delta \beta / P$, which is a power-independent quantity proportional to the strength of nonlinear effects in the fiber.

In order to compute $\gamma$, we again find the lowest-order shift $\Delta \beta$ from the perturbation Eq. (7), using (fully vectorial) perturbation theory $[15,34-36]$. This $\Delta \beta^{(1)}$ is proportional to $P$ through the field strength $|\vec{E}|^{2}$ in Eq. (7).

We now apply the above methods to compute the nonlinear strength $\gamma$ of the $\mathrm{TE}_{01}$ mode in our OmniGuide fiber, assuming that the cladding materials all have some fixed $n_{2}$. Instead of choosing a particular material $n_{2}$, we instead calculate a cladding nonlinearity suppression factor - we divide $\gamma$ by a $\gamma_{0}$, with the latter computed by supposing that both the cladding and the core have the $n_{2}$ nonlinearity. The results, plotted in Fig. 9, show that the cladding nonlinearities are suppressed by more than eight orders of magnitude over much of the bandwidth. Thus, the nonlinearities of this OmniGuide fiber will be dominated by the nonlinearities of air rather than those of the cladding, even for materials thousands of times more nonlinear than silica. Gases 


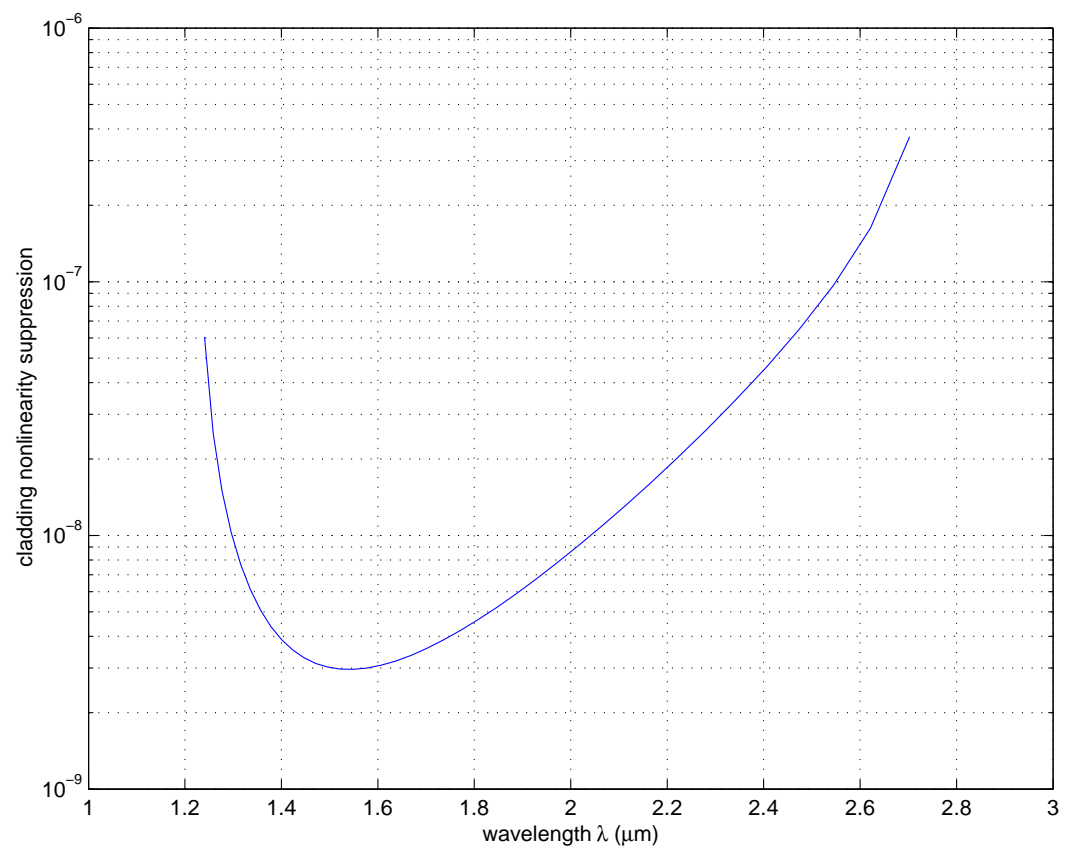

Figure 9. The $\mathrm{TE}_{01}$ mode's suppression factor for cladding nonlinearities in the OmniGuide fiber of Fig. 4, relative to nonlinearities that include the core.

have Kerr constants almost 1000 times weaker than that of silica - combined with the fact that the core area here is almost 10 times larger than the effective area of a typical silica fiber, this implies nonlinearities in the OmniGuide fiber that are almost 10,000 times weaker than those of silica fibers. Such low nonlinearities would open dramatically new areas for fiber operation: for example, high powers, closely-spaced channels and/or low/zero dispersion without regard for FWM, ${ }^{\ddagger}$ use of non return-to-zero (NRZ) formats at high bit rates, and dispersion compensation at larger intervals without regard for SPM. A better understanding of this nonlinearity suppression can be found in the scaling laws derived in the following section.

\subsection{Scaling Laws}

The scaling laws for absorption loss and nonlinearities, as a function of core radius $R$, can be derived straightforwardly from the results in Sec. 3. In particular, the perturbation-theory integral that determines the absorption loss has an integrand proportional to the fraction of $|\vec{E}|^{2}$ in the cladding [15], which scales as $1 / R^{3}$ from Eq. (3), so:

$$
\mathrm{TE}_{0 \ell} \text { cladding absorption } \sim \frac{1}{R^{3}} .
$$

This is a familiar result, since it is the same as the scaling of the $\mathrm{TE}_{01}$ ohmic dissipation losses in a hollow metallic waveguide. The scaling for the nonlinear strength $\gamma$ is found by similar arguments. Here, however, there is an additional factor of $|\vec{E}|^{2}$ from Eq. (7), and thus $1 / R^{4}$ from Eq. (2). The nonlinear strength $\gamma$ of the cladding therefore scales like $1 / R^{7}$ ! The nonlinear strength $\gamma_{0}$, when one adds nonlinearities to the core, scales inversely with the area $R^{2}$ as in an ordinary fiber, so:

$$
\mathrm{TE}_{0 \ell} \text { cladding nonlinearity } \frac{\gamma}{\gamma_{0}} \sim \frac{1}{R^{5}} .
$$

${ }^{\ddagger} \mathrm{FWM}$ noise is proportional to $\gamma^{2} /\left(\Delta \beta^{2}+\alpha^{2}\right)$, where the $\alpha^{2}$ term is usually negligible [18]. However, with nonlinearities 10,000 times weaker than in silica, $\alpha=0.01 \mathrm{~dB} / \mathrm{km}$ is sufficient to suppress FWM even in the limit of $\Delta \beta=0$ (zero dispersion and/or channel spacing). 


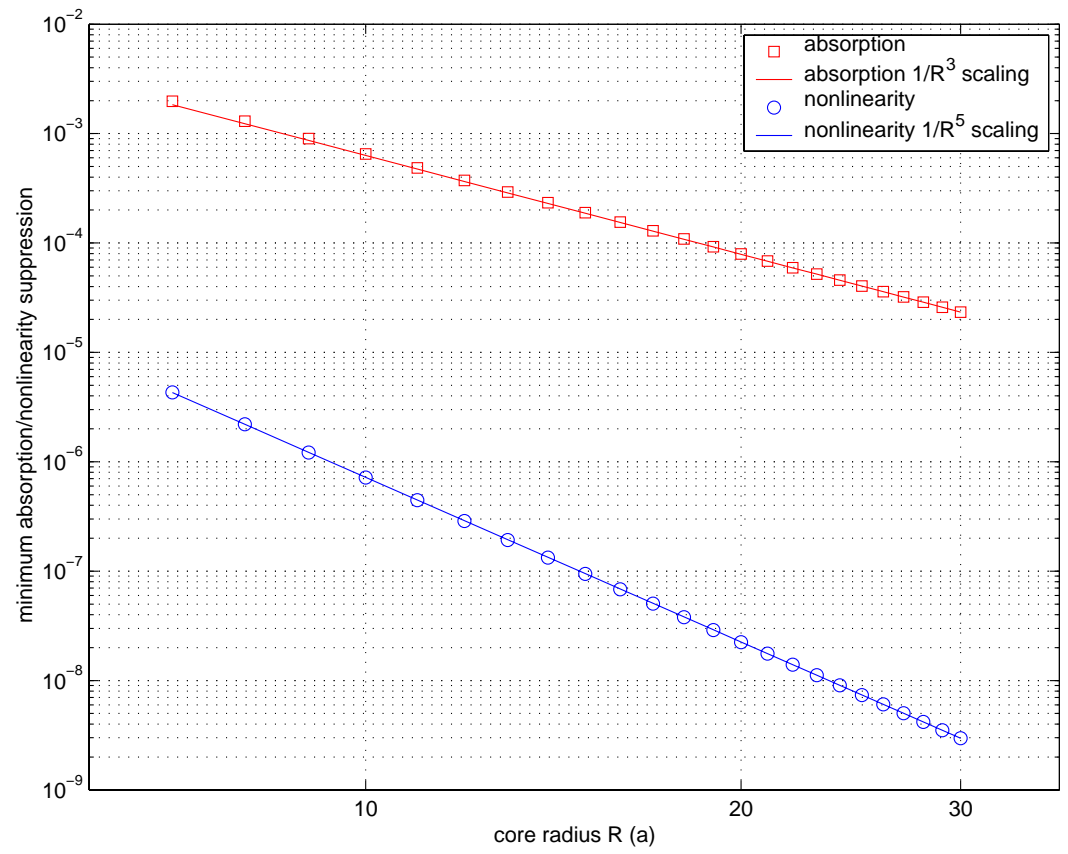

Figure 10. Scaling of the cladding absorption and nonlinearity suppression factors for a core radius $R$ varying from $7 a$ to $30 a$ (taking the minimum over the $\mathrm{TE}_{01}$ band at each radius). Hollow squares/circles show the computed values, and the solid lines display the values predicted by starting from the $30 a$ value and applying the scaling laws.

It is because of these rapid $1 / R^{3}$ and $1 / R^{5}$ scalings that the cladding absorption and nonlinearities can be suppressed so strongly for $\mathrm{TE}_{01}$ in a large-core OmniGuide fiber. To demonstrate these scaling laws explicitly, we plot the absorption and nonlinear suppression coefficients as a function of $R$ in Fig. 10, superimposing the predicted scaling laws.

\section{CONCLUDING REMARKS}

We have presented the propagation characteristics of the lowest-loss $\mathrm{TE}_{01}$ mode in an example OmniGuide fiber with a large core radius $R=30 a$. We have demonstrated that the material absorption and nonlinearities of the cladding are suppressed by four or more orders of magnitude, holding forth the promise of optical fibers with substantially lower losses and negligible nonlinearities compared to conventional silica fibers - even when highly lossy and nonlinear materials are used for the multilayer cladding. (The same properties open the possibility of fibers for currently inaccessible wavelengths.) The fiber properties are dominated by the geometry of the core, and for a wide range of $R$ follow generalized scaling relations that we have derived. Even though such a large-core OmniGuide fiber is ostensibly highly multi-mode, the higher losses of the other modes creates a modal-filtering effect that allows the fiber to operate in a single-mode fashion as long as the differential losses are greater than the rate of coupling from imperfections. In this respect, and in many others, such a fiber is analogous to the hollow metallic waveguides that have been extensively studied in the microwave regime. Finally, since the lowest-loss $\mathrm{TE}_{01}$ mode is non-degenerate, it is immune to the polarization-mode dispersion (PMD) effects that can limit bit rates in silica fibers. In future publications, we will consider many of these issues in greater detail, as well as other topics such as input/output coupling and dispersion tailoring. 


\section{REFERENCES}

1. J. D. Joannopoulos, R. D. Meade, and J. N. Winn, Photonic Crystals: Molding the Flow of Light, Princeton, 1995.

2. R. F. Cregan, B. J. Mangan, J. C. Knight, T. A. Birks, P. S.-J. Russell, and P. J. Roberts, "Single-mode photonic band gap guidance of light in air," Science 285, pp. 1537-1539, 1999.

3. P. Yeh, A. Yariv, and E. Marom, "Theory of Bragg fiber," J. Opt. Soc. Am. 68, pp. 1196-1201, 1978.

4. N. J. Doran and K. J. Bulow, "Cylindrical Bragg fibers: a design and feasibility study for optical communications," J. Lightwave Tech. 1, pp. 588-590, 1983.

5. A. N. Lazarchik, "Bragg fiber lightguides," Radiotekhnika i electronika 1, pp. 36-43, 1988.

6. C. M. de Sterke and I. M. Bassett, "Differential losses in Bragg fibers," J. Appl. Phys. 76, pp. 680-688, 1994.

7. Y. Fink, D. J. Ripin, S. Fan, C. Chen, J. D. Joannopoulos, and E. L. Thomas, "Guiding optical light in air using an all-dielectric structure," J. Lightwave Tech. 17, pp. 2039-2041, 1999.

8. F. Brechet, P. Roy, J. Marcou, and D. Pagnoux, "Singlemode propagation into depressed-core-index photonic-bandgap fibre designed for zero-dispersion propagation at short wavelengths," Elec. Lett. 36(6), pp. 514-515, 2000.

9. F. Brechet, P. Leproux, P. Roy, J. Marcou, and D. Pagnoux, "Analysis of bandpass filtering behavior of singlemode depressed-core-index photonic bandgap fibre," Elec. Lett. 36(10), pp. 870-872, 2000.

10. M. Ibanescu, Y. Fink, S. Fan, E. L. Thomas, and J. D. Joannopoulos, "An all-dielectric coaxial waveguide," Science 289, pp. 415-419, 2000.

11. Y. Xu, R. K. Lee, and A. Yariv, "Asymptotic analysis of Bragg fibers," Opt. Lett. 25, pp. 1756-1758, 2000.

12. T. Kawanishi and M. Izutsu, "Coaxial periodic optical waveguide," Opt. Express 7(1), pp. 10-22, 2000. http://www.opticsexpress.org/oearchive/source/22933.htm.

13. Y. Xu and A. Yariv, "Asymptotic analysis of Bragg fibers and dielectric coaxial fibers," in Proc. SPIE, A. Dutta, A. A. S. Awwal, N. K. Dutta, and K. Okamoto, eds., 4532, pp. 191-205, 2001.

14. G. Ouyang, Y. Xu, and A. Yariv, "Comparative study of air-core and coaxial Bragg fibers: single-mode transmission and dispersion characteristics," Opt. Express 9(13), pp. 733-747, 2001. http://www.opticsexpress.org/oearchive/source/37552.htm.

15. S. G. Johnson, M. Ibanescu, M. Skorobogatiy, O. Weisberg, T. D. Engeness, M. Soljačić, S. A. Jacobs, J. D. Joannopoulos, and Y. Fink, "Low-loss asymptotically single-mode propagation in large-core OmniGuide fibers," Opt. Express 9(13), pp. 748-779, 2001. http://www.opticsexpress.org/oearchive/source/37483.htm.

16. M. Ibanescu, S. G. Johnson, M. Soljačić, J. D. Joannopoulos, Y. Fink, O. Weisberg, T. D. Engeness, S. A. Jacobs, and M. Skorobogatiy, "Analysis of mode structure in OmniGuide fibers," Phys. Rev. B , 2002. Submitted for publication.

17. Y. Fink, J. N. Winn, S. Fan, C. Chen, J. Michel, J. D. Joannopoulos, and E. L. Thomas, "A dielectric omnidirectional reflector," Science 282, pp. 1679-1682, 1998.

18. R. Ramaswami and K. N. Sivarajan, Optical Networks: A Practical Perspective, Academic Press, London, London, 1998.

19. E. A. Marcatili and R. A. Schmeltzer, "Hollow metallic and dielectric waveguides for long distance optical transmission and lasers," Bell Syst. Tech. J. 43, pp. 1783-1809, 1964.

20. W. D. Warters, "WT4 millimeter waveguide system: introduction," Bell Syst. Tech. J. 56, pp. 1825-1827, 1977. The introduction to a special issue with many useful articles.

21. M. Miyagi, A. Hongo, and S. Kawakami, "Transmission characteristics of dielectric-coated metallic waveguides for infrared transmission: slab waveguide model," IEEE J. Quantum Elec. QE-19(2), pp. 136-145, 1983.

22. M. Miyagi and S. Kawakami, "Design theory of dielectric-coated circular metallic waveguides for infrared transmission," J. Lightwave Tech. 2, pp. 116-126, 1984.

23. J. A. Harrington, "A review of IR transmitting, hollow waveguides," Fiber Integr. Opt. 19, pp. 211-227, 2000.

24. P. Yeh, Optical Waves in Layered Media, Wiley, New York, 1988. 
25. A. W. Snyder and J. D. Love, Optical Waveguide Theory, Chapman and Hall, London, 1983.

26. S. A. Jacobs and al., "Radiation loss and leaky modes in depressed-core waveguides." To be published in 2002.

27. L. Grüner-Nielsen, S. N. Knudsen, B. Edvold, T. Veng, D. Magnussen, C. C. Larsen, and H. Damsgaard, "Dispersion compensating fibers," Optical Fiber Tech. 6, pp. 164-180, 2000.

28. W. H. Weber, S. L. McCarthy, and G. W. Ford, "Perturbation theory applied to gain or loss in an optical waveguide," Appl. Opt. 13(4), pp. 715-716, 1974.

29. A. Kumar, S. I. Hosain, and A. K. Ghatak, "Propagation characteristics of weakly guiding lossy fibers: an exact and perturbation analysis," Optica Acta 28(4), pp. 559-566, 1981.

30. Z. Pantic and R. Mittra, "Quasi-TEM analysis of microwave transmission lines by the finite-element method," IEEE Trans. Microwave Theory Tech. MTT-34(11), pp. 1096-1103, 1986.

31. S. X. She, "Propagation loss in metal-clad waveguides and weakly absorptive waveguides by a perturbation method," Opt. Lett. 15(16), pp. 900-902, 1990.

32. V. L. Gupta and E. K. Sharma, "Metal-clad and absorptive multilayer waveguides: an accurate perturbation analysis," J. Opt. Soc. Am. A 9(6), pp. 953-956, 1992.

33. C. Themistos, B. M. A. Rahman, A. Hadjicharalambous, and K. T. V. Grattan, "Loss/gain characterization of optical waveguides," J. Lightwave Tech. 13(8), pp. 1760-1765, 1995.

34. D. Sarid and G. I. Stegeman, "Optimization of the effects of power dependent refractive indices in optical waveguides," J. Appl. Phys. 52(9), pp. 5439-5441, 1981.

35. V. P. Tzolov, M. Fontaine, N. Godbout, and S. Lacroix, "Nonlinear self-phase-modulation effects: a vectorial first-order perturbation approach," Opt. Lett. 20(5), pp. 456-458, 1995.

36. R. S. Grant, "Effective non-linear coefficients in optical waveguides," Optical and Quantum Elec. 28, pp. 1161-1173, 1996. 